
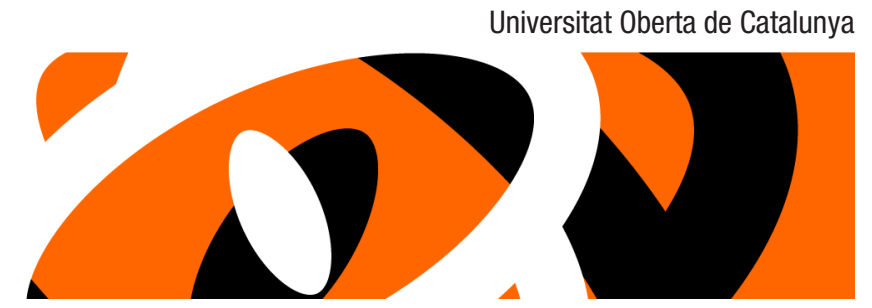

http://artnodes.uoc.edu

\title{
Prácticas artísticas en la época del dataceno Data Biography: rastros digitales para la exploración biográfica de la identidad personal*
}

\author{
Diego Díaz \\ Universitat Juame I
}

\section{Clara Boj}

Universidad Politécnica de Valencia

Fecha de presentación: marzo de 2019

Fecha de aceptación: mayo de 2019

Fecha de publicación: julio de 2019

\section{Cita recomendada}

Díaz, Diego; Boj, Clara. 2019. «Prácticas artísticas en la época del dataceno. Data Biography. rastros digitales para la exploración biográfica de la identidad personal». Artnodes. N. ${ }^{\circ} 24$ : 121-133. UOC [Fecha de consulta: dd/mm/aa]. http://dx.doi.org/10.7238/a.v0i24.3293

\footnotetext{
* Este proyecto se está realizado en el contexto del proyecto de investigación «Tecnologías de juegos para la creación de contenidos digitales, gestión, visualización y sonificación, tin2016-75866-c3-1-r», financiado por el MINECO.
} 


\title{
artnodes
}

http://artnodes.uoc.edu

Prácticas artísticas en la época del dataceno...

\section{Resumen}

Cada vez más detalles de nuestra vida son registrados y almacenados para formar parte de lo que ha venido a llamarse big data. Nuestra vida, en todos sus aspectos, deja un rastro digital que se actualiza continuamente en función de actividades, tanto de la vida física como de la vida digital, que alimentan bases de datos en las que se refleja tanto el último libro que hemos comprado como las visitas médicas que hemos realizado. Muchos de esos datos son abiertos y accesibles, otros son propiedad de empresas privadas que comercializan con ellos.

En esta publicación inicialmente introducimos y justificamos la conveniencia de utilizar el termino dataceno, entendido como una metáfora para comprender la sociedad actual como una sociedad mediada por los datos digitales. Posteriormente, realizamos una clasificación de las practicas artísticas contemporáneas con distintas aproximaciones al uso y análisis de datos complejos: desde la creación de atractivas visualizaciones bajo el propósito de acceder a la información escondida en los grandes volúmenes de datos, hasta aproximaciones más conceptuales que nos acercan a un planteamiento crítico sobre el volumen, recolección, uso y comercialización de los mismos.

En la última parte de esta publicación, nos centramos en analizar las creaciones artísticas basadas en rastros digitales y en el concepto de biografía narrativa en la era del dataceno. Por último, analizamos nuestra obra Data Biography, una propuesta de diseño especulativo que cuestiona la escritura biográfica, utilizando los datos recogidos de nuestro rastro digital en redes sociales, WhatsApp, correos electrónicos, sitios web visitados, búsquedas en Google, imágenes, ubicación de gps, etc.

\section{Palabras clave}

big data, biografía de datos, dataceno, arte de los medios, rastros digitales

\section{Artistic practices in the age of the datacene \\ Data Biography: digital traces to biographically explore personal identity}

\begin{abstract}
More and more details of our lives are recorded and stored as part of what has been called big data. Our lives, in all their aspects, leave a digital trail that is continually updated in terms of activities. Our physical and digital lives are recorded in databases with the latest book we purchased and with our medical visits. Many of these data are open and accessible, others are owned by private companies that use them for marketing purposes.

In this publication, we provide an initial introduction and justification of the usefulness of the term datacene understood as a metaphor to comprehend our current society mediated by digital data. Then we establish a classification of the contemporary artistic practices with different approaches to the use and analysis of complex data; from the creation of aestheticallypleasing visualizations of large volumes of data, to more conceptual approaches that critically analyze the volume, collection, use and marketing of data.

In the last part of this publication we focus on artistic creations based on digital traces and the concept of narrative biography in the age of the datacene where we present our work Data Biography; a speculative design proposal that questions how we could write a current individual's biography using the data captured from our digital trail on social networks, WhatsApp, emails, websites visited, Google searches, images, GPS location, and so forth.
\end{abstract}

\section{Keywords}

big data, data biography, datacene, media art, digital trails 


\section{artnodes}

\section{Introducción}

Una parte de la comunidad científica ha propuesto el término antropoceno para designar una nueva época en el tiempo geológico (Zalasiewicz et al. 2011), caracterizada por la dominación global del ser humano en el planeta. Este término, que goza de gran popularidad en las investigaciones académicas actuales, se ha convertido también en un fenómeno global que se ha expandido desde el ámbito académico al pensamiento cultural, social y político más amplio (Malhi 2017). E antropoceno representa en términos generales cómo los humanos hemos llegado a ser la fuerza dominante que moldea el medio ambiente de la Tierra, con devastadoras consecuencias para nuestra propia supervivencia y la de otras especies. Si desde la geología, el antropoceno designa el inicio de un nuevo periodo temporal en la historia de la vida del planeta, desde las humanidades en general esta nomenclatura se utiliza como metáfora paraguas que hace referencia al calado y velocidad de las transformaciones que afectan a las sociedades contemporáneas, desde perspectivas culturales, políticas y tecnológicas, y cómo estas dan forma al desarrollo de la vida en el planeta.

Muestra de la gran popularidad del término es la cantidad de nomenclatura asociada aparecida en los últimos años que, utilizando el símil del tiempo geológico, apunta a momentos de la historia que se definen y modelan a través de los efectos que determinados sistemas, herramientas o pensamientos producen sobre la existencia.

Así, diversos autores hablan del capitaloceno para definir una época desde la perspectiva del sistema económico bajo el que se desarrolla, fundamentalmente marcado por la dominación del capital (Moore 2016). Otros apuntan al homogenoceno para definir la homogeneización de la vida biológica después de la reintegración física de los ecosistemas, que alguna vez fueron aislados, de las Américas, Eurasia y África a finales del siglo xv (Curnutt 2007; Samways 1999). También Dona Haraway (Haraway 2015) hace uso del sufijo «ceno» para proponer Chthulucene como el desarrollo de un tiempo futuro marcado por la empatía entre las especies.

En este ensayo proponemos el término dataceno, entendido como un marco de reflexión de la época actual a partir del desarrollo del big data y su incidencia de manera directa en el desarrollo de las estructuras sociales, culturales, económicas, políticas e incluso afectivas del presente.

Aunque el almacenamiento, registro y procesado de cantidades masivas de datos no es algo reciente ni exclusivo de esta época, la evolución de las tecnologías digitales y la computación ha propiciado un avance exponencial en los sistemas de registro y captación de datos, así como en su almacenamiento y análisis, dando paso al big data. Este concepto rápidamente asimilado define un sistema conceptual e instrumental reciente que marca un hito importante en la historia de la evolución humana, puesto que sus consecuencias no solo son evidentes en la cultura, la economía y la política, sino también en otras áreas tan diversas como la sanidad o el arte.
Cuando hablamos del dataceno no proponemos una nueva era geológica, ya que no queda marcada en el estratotipo, pero en la historia de la evolución humana sí que está marcando un cambio importante, ya que está suponiendo una verdadera revolución de conocimiento, al desafiar las epistemologías establecidas en las ciencias, las ciencias sociales y las humanidades, y generar cambios de paradigma en múltiples disciplinas. De la mano del big data están surgiendo nuevas formas de empirismo que declaran «el fin de la teoría», la creación de ciencia basada en datos en lugar de conocimiento, y el desarrollo de humanidades digitales y ciencias sociales computacionales que proponen formas radicalmente diferentes de tener sentido de cultura, historia, economía y sociedad (Kitchin 2014).

Bajo la perspectiva del dataceno, en este artículo vamos a analizar cómo este espacio de información masiva puede ser un territorio de experimentación artística y en concreto para la exploración biográfica de la identidad personal.

\section{Dataceno: la era de los datos}

Estamos asistiendo al desarrollado de una verdadera revolución de los datos digitales, donde los ecosistemas han dejado de ser exclusivamente materiales para convertirse en sistemas híbridos, físico-digitales. Los bits y los átomos se entremezclan en una especie de átomos radicales (Radical Atom) (Ishii et al. 2012), en la que los elementos físicos tienen su correlación digital, generados (entre otros) por las redes sociales, las ciudades inteligentes y el internet de las cosas (loT, por sus siglas en inglés) (Jucevicius, Patasienè y Patasius 2014; Albino, Berardi, y Dangelico 2015). Pero el verdadero protagonista de este espacio híbrido que compone el dataceno son Ios datos masivamente generados y que se ha venido a denominar big data (Mashey 1998), en referencia a los grandes conjuntos de datos en constante crecimiento con tres características principales, las denominadas 3V:Volumen, Velocidad y Variedad (Furht y Villanustre 2016). Según un reciente informe de International Digital Corporation, para el año 2025 se alcanzará la increíble cantidad de 163 zetabytes (IDC 2018) de datos generados por millones de dispositivos y aplicaciones. Una buena parte de ellos estarán formados por nuestras huellas digitales, consistentes en datos provenientes del registro de nuestra actividad cotidiana en el entorno físico y digital. Según Weaver y Gahegan (2010), podemos clasificar estas huellas digitales en pasivas, recopiladas sin que el propietario lo sepa, y en activas, creadas cuando los datos personales son liberados a propósito por un usuario con el fin de compartir información sobre uno mismo a través de sitios web o redes sociales.

Uno de los mayores ámbitos de desarrollo del dataceno es el Business Data Analytics (BDA) ó análisis de datos de negocio, con una tasa de crecimiento anual del $11,9 \%$ entre los años 2017 y 2022, y que principalmente se aplica a la banca, la industria de fabricación 


\section{artnodes}

http://artnodes.uoc.edu

Prácticas artísticas en la época del dataceno...

discreta y de procesos, los servicios profesionales y el gobierno (IDC 2018). Pero el big data se está utilizando actualmente en otros muchos ámbitos, al recolectar continuamente multitud de datos provenientes de diversos dispositivos y aplicaciones, que podemos clasificar en base a las características que podemos ver en la figura 1.

En este contexto de generación masiva de datos podemos entender el dataceno como una metáfora de la sociedad mediada por los datos, en la que se registra, almacena y analiza una inmensidad de datos heterogéneos. Estos datos, unidos al increíble potencial de las nuevas técnicas de inteligencia artificial que utilizan el big data como apoyo para el funcionamiento de los algoritmos predictivos y el machine learning, están transformando de manera significativa nuestras sociedades. Como apunta (Danaher 2016), el uso de las decisiones basadas en algoritmos en la esfera pública y política puede ser problemático, debido a que los actuales algoritmos de machine learning no responden a una estructura secuenciada y predecible de órdenes, sino que su funcionamiento interno es opaco, autónomo y actúa como una caja negra. Aunque entrar en un análisis de estas implicaciones escapa a los límites de esta publicación, sí nos gustaría resaltar que estos algoritmos necesitan amplios conjuntos de datos 0 datasets para entrenar su funcionamiento. Por lo tanto, el valor de estos algoritmos recae principalmente en los conjuntos de datos (datasets) utilizados para su entrenamiento, ya que en ellos recae su buen funcionamiento. Entendemos, pues, los datos como el nuevo oro de la sociedad actual, y este es uno de los motivos por los que creemos pertinente utilizar el termino dataceno.

Por otro lado, la infraestructura física que da soporte al dataceno está formada por una red distribuida e interconectada a escala planetaria de servidores físicos ubicados en centros de datos (data centers), además de la multitud de dispositivos electrónicos que recopila y envía los datos a estos servidores. Podemos entender que esta red de centros de datos funciona como un inmenso archivo de la humanidad, una inmensa biblioteca digital que guarda nuestra historia reciente, la historia del dataceno. Para tener una magnitud de referencia sobre el impacto de estos data centers, podemos mencionar que consumen actualmente en torno al $2 \%$ de la energía mundial. Pero además, para recolectar los datos y enviarlos, utilizamos multitud de dispositivos electrónicos, que consumen alrededor del $5 \%$ de la energía global. Se calcula que para el año 2025, debido al constante aumento en el número y potencia de estos dispositivos, utilizarán el $20 \%$ de toda la electricidad del mundo y emitirán hasta el 5,5\% de todas las emisiones de carbono, por no citar los enormes requerimientos en energía y materias primas necesarios para su reciclado y fabricación.

Como podemos ver, la sociedad del dataceno tiene un gran reto ecológico y energético, al estar compuesta por un torrente en continuo aumento de dispositivos conectados a internet, fotos de alta resolución, vídeos transmitidos por correo electrónico, secuencias de vídeo de cámaras de vigilancia, teléfonos y televisores inteligentes, etc., que al aumentar constantemente en número, necesitan cada vez más recursos energéticos y de materias primas. Además, el aumento de su potencia de cómputo y definición requieren mayor cantidad de ancho de banda y cantidades cada vez mayores de almacenamiento.

Debido a los límites e intereses de esta publicación, no nos planteamos realizar una exhaustiva justificación del termino dataceno y los problemas estructurales que plantea, así que dejamos esta tarea para futuras publicaciones. En este artículo nos centraremos en realizar un análisis de distintas propuestas artísticas relacionadas con el dataceno, para centrarnos en aquellas vinculadas con el género biográfico. Nuestro objetivo es centrarnos en el uso de los datos digitales como recurso para generar biografías de datos y los aspectos críticos asociados a esta tarea, relacionados con la carencia de privacidad y los posibles errores en las técnicas de predicción de

Figura 1. Tabla de clasificación de dispositivos y aplicaciones del big data

\begin{tabular}{|c|c|c|c|}
\hline denominación & dispositivo & tipo de dato & Aplicaciones \\
\hline $\begin{array}{l}\text { internet de las cosas } \\
\text { Smart Grid }\end{array}$ & $\begin{array}{l}\text { red de sensores inteligentes } \\
\text { distribuidos y embebidos en la } \\
\text { ciudad o el medio rural }\end{array}$ & $\begin{array}{l}\text { multiple: ubicación, } \\
\text { posicionamiento, estado, } \\
\text { funcionamiento, etc. }\end{array}$ & $\begin{array}{l}\text { seguridad, mantenimiento, } \\
\text { optimización de recursos (redes de } \\
\text { tranporte, eléctrica, agua potable, } \\
\text { agricultura, etc.) (Diamantoulakis, } \\
\text { Kapinas y Karagiannidis 2015) } \\
\text { (Philip Chen y Zhang 2014) } \\
\text { (Chen y Han 2018) }\end{array}$ \\
\hline e-salud y quantified self (QS) & $\begin{array}{l}\text { sensores biomédicos acoplados } \\
\text { al cuerpo }\end{array}$ & $\begin{array}{l}\text { ritmo cardiaco, presión sanguínea, } \\
\text { nivel de oxigeno y azúcar, etc. }\end{array}$ & $\begin{array}{l}\text { adaptabilidad y optimización de } \\
\text { los planes de salud y el ADN (Asri } \\
\text { et al. 2015) }\end{array}$ \\
\hline actividad en línea & $\begin{array}{l}\text { teléfonos inteligentes, tabletas, } \\
\text { ordenadores y otros dispositivos } \\
\text { que llevamos con nosotros }\end{array}$ & $\begin{array}{l}\text { presencia digitales en redes } \\
\text { sociales, email, web, ubicación, } \\
\text { posicionamiento }\end{array}$ & $\begin{array}{l}\text { servicios políticos y monitoreo } \\
\text { gubernamental, comercio } \\
\text { electrónico (Erevelles, Fukawa y } \\
\text { Swayne 2016) (Kim, Trimi y Chung } \\
\text { 2014) }\end{array}$ \\
\hline
\end{tabular}




\section{artnodes}

http://artnodes.uoc.edu

patrones de comportamiento, aspectos que consideramos críticos en la sociedad del dataceno, donde casi todas nuestras acciones quedan registradas en el big data.

\section{Creación artística en la época del dataceno}

Un número creciente de artistas utilizan los datos digitales como materia prima para la generación de sus proyectos. En muchos casos, los usan para realizar visualizaciones bidimensionales a modo de ilustración, ya sean interactivas o estáticas, imprimibles o no. Generalmente la finalidad de estas propuestas es la de analizar y acceder a la información oculta en los datos a través de su visualización, ya sea con fines críticos o meramente estéticos y/o narrativos, o bajo una intencionalidad más heterogénea y/o conceptual.

Dentro del primer grupo, nos encontramos con un campo de experimentación artístico relativamente nuevo, que tiene sus raíces en una larga tradición histórica de representación de la información mediante imágenes que combinan el arte, la ciencia y las estadísticas (Friendly 2006). Los artistas crean, a partir de datos provenientes generalmente de las bases de datos libres (open big data), determinados algoritmos gráficos para acceder a los datos, procesarlos y generar representaciones visuales. Una de las primeras propuestas que encontramos en este sentido es un repositorio web que acumula más de mil representaciones visuales de datos, fundado por Manuel Lima, Ilamado Visual Complexity (Lima 2005), en el cual el autor ha ido recopilando desde el año 2005 ejemplos de representaciones de redes complejas con «el objetivo de aportar una comprensión crítica de los diferentes métodos de visualización, a través de una serie de disciplinas, tan dispares como la biología, las redes sociales o la world wide web» y en base a esta "compresión crítica» propone además trabajar en el desarrollo de una teoría general de la complejidad, con la intención no solo de conocer mejor nuestro mundo, sino también de conocernos mejor a nosotros mismos.

A finales de la primera década del siglo xxı nos encontramos con multitud de ejemplos desarrollados bajo la denominación data art 0 arte de los datos, como el proyecto de David McCandless Information Is Beautiful (McCandless 2009a). Su sitio web está lleno de representaciones visuales con un claro uso del diseño y una funcionalidad comunicativa, que nos permite entender de forma clara y sencilla la información escondida en los datos, o como él mismo explica en su web: «estoy interesando en cómo la información diseñada puede ayudarnos a entender el mundo, a revisar las noticias falsas y a revelar las conexiones, patrones e historias ocultas» (McCandless 2009b). Un caso parecido es el sitio web Moebio.com, donde también encontramos elaboradas representaciones visuales de datos complejos (Ortiz 2002). En la actualidad, la popular librería de JavaScript D3.js o Data-Driven Documents (D3 2011), distribuida gratuitamente bajo licencia de código libre, es utilizada masivamente para producir, a partir de datos, infogramas dinámicos e interactivos en navegadores web modernos.

Otro popular entorno de desarrollo de representaciones gráficas de datos es Processing. Iniciado por Ben Fry y Casey Reas en el 2001, también distribuido gratuitamente y de código abierto, basado en Java, goza de gran popularidad entre la comunidad artística, gracias a que fue diseñado por y para artistas. Flight Patterns, de Aaron Koblin, es un ejemplo de visualización de datos realizada con Processing, que nos muestra la saturación del espacio aéreo en Estados Unidos, al representar gráficamente la información de 24 horas de vuelos sobre el país. Esta obra establece un paralelismo con las redes neuronales, donde determinadas interconexiones son más activas que otras y donde podemos también encontrar áreas de baja o ninguna actividad. Flight Patterns (Koblin 2009) no solo nos permite visualizar de manera objetiva y directa la desmesurada cantidad de vuelos que diariamente sobrevuelan Estados Unidos, sino que además nos invita a reflexionar sobre su sentido y necesidad y los efectos que estos producen a escala económica, social y ecológica.

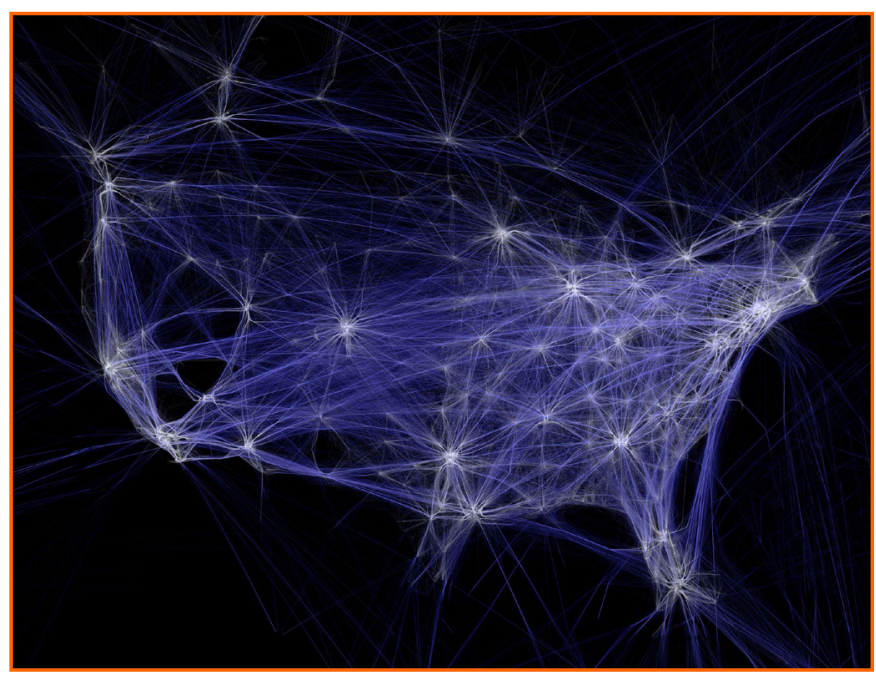

Imagen 1. Flight Patterns, Aaron Koblin, 2009

Bajo una perspectiva más heterogénea y no tan enfocada en el objetivo de mostrar la información de una manera atractiva y accesible, sino en un tratamiento más cercano al arte político y conceptual, podemos encontrar CarnivorePE, del colectivo Radical Software Group, iniciado en el año 2001. Se trata de un software de "edición privada» basado en el Carnivore del FBI (el apodo del DS1000 utilizado por el FBI para el rastreo de datos). CarnivorePE fue construido para brindar a los artistas la oportunidad de explorar el rastreo de datos como una forma de arte y aportar una visión crítica sobre los límites de la vigilancia gubernamental en la era digital y la continuada violación de la privacidad por empresas y estados. CarnivorePE capta la información transmitida en las redes de comunicación (concretamente el protocolo $\mathrm{TCP} / \mathrm{IP}$ ) y la reenvía a los distintos clientes, desarrollados por artistas 


\section{artnodes}

http://artnodes.uoc.edu

Prácticas artísticas en la época del dataceno...

y colectivos desde diversas perspectivas. Uno de estos proyectos fue «Red Libre Red Visible» (Diaz et al. 2005) en el cual desarrollamos una aplicación de realidad aumentada que realizaba una visualización a tiempo real de los datos captados por el CarnivorePE, ubicada en el espacio físico con la intención de mostrar la conquista del espacio físico por los datos digitales.

Siguiendo con esta línea de investigación, unos años después realizamos el proyecto «Observatorio» (Boj et al. 2010) en el que unos dispositivos a modo de binoculares turísticos, ubicados en las atalayas de distintas ciudades (Valencia, Gijón, Karlsruhe, Tallinn y Barcelona), rastrearon el espectro de las redes inalámbricas con el objetivo de cartografíar el paisaje de las ciudades híbridas. Este proyecto participó, entre otras exposiciones, en «Invisible Fields» (De Vicente, Harger y Perelló 2011), donde se mostraron un buen numero de proyectos que exploraron cómo la comprensión de nuestro mundo y nuestro cosmos se ha transformado por el desarrollo de las ondas de radiofrecuencia y cómo en la actualidad las ondas son el canal de comunicación de los datos digitales.

Unos años después, en la exposición temática sobre los datos Big Bang Data (De Vicente y Subirós 2014), podemos encontrar una amplia selección de trabajos seleccionados desde distintos ejes, pero bajo el planteamiento general del análisis crítico y representación, ya sea física, sonora o visual, de los datos y su complejidad. Uno de los proyectos que participaron en esta exposición fue «HRs in photos» de Erik Kessels (KesselsKramer 2011), una instalación formada por un mar de fotografías impresas que corresponden a las imágenes subidas a Flickr en un periodo de 24 horas, donde el hecho de imprimirlas nos da una visión física abrumadora de lo que significa este más de un millón de imágenes. Este proyecto nació con el propósito de mostrar el diluvio de imágenes que internet condensa diariamente y cómo nos hemos habituado a consumirlas, así como de tomar conciencia del hecho de que las fotografías pasan del ámbito privado al público al subirlas a la red.

Con «HRs in photos» nos encontramos ante una instalación que transforma en elementos físicos la información digital, desde

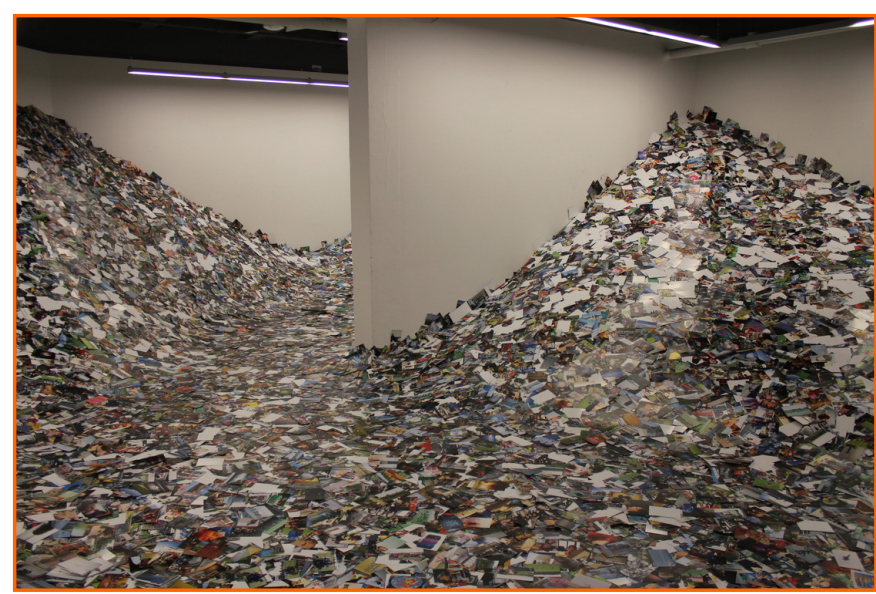

Imagen 2. «HRs in photos», Erik Kessels, 2011 una perspectiva similar a la denominada fisicalización de datos 0 visualización física, una practica basada en una reciente área de investigación, consistente en la representación física de los datos, gracias a los avances en la fabricación digital, las interfaces tangibles activas y las pantallas que cambian de forma (Sosa et al. 2018). De manera similar a la visualización de datos, la fisicalización de datos tiene como objetivo ayudar a las personas a explorar, comprender y comunicar sistemas de datos complejos, pero en este caso mediante la representación física, no digital, de los mismos.

\section{Creación artística y datos personales: reflejos del yo en el dataceno}

En nuestra actividad diaria, tanto física como digital, de manera consciente e inconsciente, generamos una enorme cantidad de rastros digitales que son almacenados en distintas bases de datos. Nuestros dispositivos digitales, principalmente el teléfono inteligente, pero también pulseras, relojes electrónicos y otros, se han convertido en una suerte de «chivato expiatorio», que comparte datos y metadatos de nuestra actividad digital. A continuación, en la figura 2, presentamos una clasificación del tipo de rastros digitales, los dispositivos que los generan y el nivel de consciencia que tenemos de haberlos generado, basada en los estudios de Balebako et al. 2013; Graeff y Harmon 2002; Schwartz 2004; Colombo y Ferrari 2015; Smith et al. 2012. Como podemos observar en la clasificación anterior, existe una gran variedad de situaciones en las que, diariamente y de forma casi continua, vamos generando datos digitales que hacen crecer el big data. En algunos casos, los usuarios pueden descargar parte de la información que las empresas tienen sobre ellos. Por ejemplo, Google, Facebook y Twitter, entre otros, directamente te ofrecen esta opción en su espacio web, ya que, de acuerdo con la legislación europea de protección de datos, todo individuo tiene derecho a recibir una copia de toda la información personal que una compañía ha recogido sobre él. En «Europe vs Facebook», Max Schrems (2011) quiso poner a prueba la aplicación práctica de este derecho, reclamando a Facebook Ireland Limited una copia de todas las interacciones que había realizado a través de su cuenta. Facebook le remitió un documento PDF de 1200 páginas, en el cual figuran desde transcripciones de chats hasta solicitudes de amistad denegadas.

Esta multitud de interacciones y el valor de la posesión de las mismas es el tema central de la obra Data Shop, creada por el duo artístico formado por Varvara Guljajeva and Mar Canet en el año 2017 (Guljajeva y Canet 2017). En este proyecto, nos encontramos con una tienda de latas de conserva que, en su interior, contienen memorias USB con los datos digitales extraídos de Facebook, Google Takeout, Visa y Mastercard. El proyecto propone una reflexión sobre cómo nuestros datos digitales se recopilan, utilizan, venden, roban e intercambian constantemente. Otro ejemplo en este sentido, pero 


\section{artnodes}

http://artnodes.uoc.edu

Prácticas artísticas en la época del dataceno...

Figura 2. Tabla de clasificación de rastros digitales

$\begin{array}{ll}\text { Tipo de dato Caracteristicas técnicas (val } \\ \text { ubicación } & \begin{array}{l}\text { valor GPS (latitud y longitud), } \\ \text { tiempo }\end{array}\end{array}$
tiempo

conversaciones string de texto, tiempo, ubicación GPS (en algunos casos)

sonidos, imágenes y videos, tiempo, ubicación GPS

multimedia: sonido, imágenes y vídeos
Dispositivos y aplicaciones que lo generan

teléfono móvil, pulseras de actividad y dispositivos GPS deportivos, cámaras digitales, rastreadores GPS antiperdida y robo, otros...

cualquier aplicación de comunicación para teléfono, ordenador y otros dispositivos teléfonos móviles, cámaras, ordenadores y otros dispositivos
Nivel de consciencia de generar el rastro digital (alto, medio, bajo)

Medio. Aunque los dispositivos (especialmente teléfonos inteligentes) nos preguntan si permitimos compartir nuestra ubicación, con el paso del tiempo se nos olvida esta decisión y el nivel de consciencia desciende.

Bajo. No somos conscientes de que nuestras conversaciones son almacenadas en los servidores de la empresa que nos ofrece el servicio.

medio, ya que activamos la copia de seguridad de estos contenidos en los servidores de la empresa que nos ofrece el servicio, pero con el paso del tiempo se nos olvida.

Bajo. No somos conscientes de que la información de la compra es registrada en los servidores de la empresa que vende el articulo y también de los intermediarios (bancos, paypal).

aplicaciones de teléfonos móviles y ordenadores metadatos de la compra (importe, artículo comprado, ubicación GPS tiempo)
Bajo. No somos conscientes de que la información de la compra es registrada en los servidores del banco que nos proporciona el medio de pago.

Bajo. No somos conscientes de que la información del acceso y salida es registrada en los servidores de la empresa de transporte.

Bajo. No somos conscientes de que la información del acceso y salida es registrada en los servidores de la empresa 0 institución que gestiona dicha instalación. uso de instalaciones públicas o privadas metadato del registro de entrada y salida de la instalación, tiempo, ubicación registro de acceso y salida a la instalación con tarjeta personal medio de transporte con tarjeta personal y salida al medio del transporte, tiempo, ubicación pago con tarjeta de crédito 0 teléfono móvil centrado exclusivamente en el tratamiento de los registros de nuestra ubicación, es la aplicación Frickbits (Frick and Thirteen23 2014), que propone transformar los datos de nuestra ubicación GPS en representaciones gráficas que nos muestran de forma atractiva y con un determinado valor gráfico-pictórico los recorridos y ubicaciones registradas.

De forma similar, pero aplicada al uso de imágenes, la serie de autorretratos Internet Cache Self Portrait (Evan Roth 2012) está compuesta por flujos de imágenes sin censura, recopiladas masivamente a través de la navegación diaria en Internet. «Las caras de los "amigos" de las redes sociales coexisten con los logotipos corporativos, las piezas destrozadas de los mapas de Google, las fotos familiares y los anuncios de pancartas. Estas impresiones producidas por algoritmos actúan como un desnudo contemporáneo, que expone de manera generosa y abierta las interacciones privadas en línea de los sujetos» (Roth 2012).

Pero la recopilación de datos y la posesión de los mismos es solo una parte del dataceno. Otro de los grandes campos de aplicación es la predicción de patrones y comportamientos, utilizando algoritmos predictivos en base al histórico de nuestra actividad. Este es el concepto principal de la obra / cannot not communicate de Martin Jhon Callanan (Callanan 2015). Compuesta por una biblioteca de los primeros 100 libros recomendados por Amazon en base a todo lo que el autor compró previamente en esta tienda en línea, pone en evidencia que Amazon utiliza un sofisticado algoritmo predictivo para recomendarnos futuras compras. 


\section{artnodes}

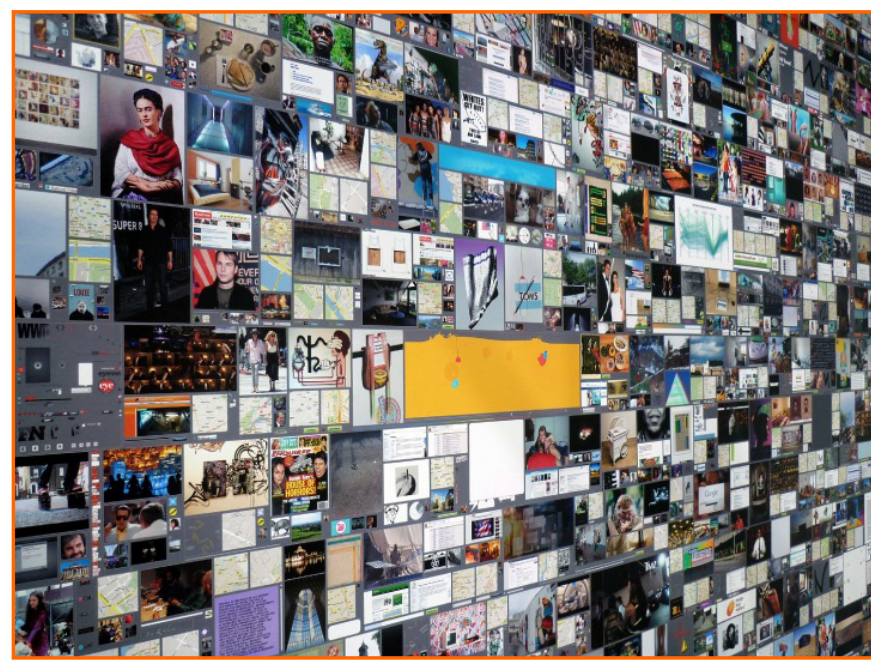

Imagen 3. Internet Cache Self Portrait, Evan Roth, 2012

Data Selfie (Do Thi Duc et al. 2016), una obra en forma de una extensión para el navegador Chrome, recolecta los datos de uso que Facebook recoge del usuario y realiza un análisis predictivo en base a esos mismos datos, intentando simular ese análisis de la forma más cercana posible a como Facebook lo hace. Los datos recopilados por Data Selfie se empaquetan de forma anónima y se envían a servidores de aprendizaje mecánico, como Watson de IBM y Apply Magic Sauce de la Universidad de Cambridge. Estas máquinas permiten generar información, como por ejemplo el tono con el que escribes comentarios 0 actualizaciones, valores, gustos 0 incluso un perfil psicológico y demográfico basado en tu actividad. En palabras de los autores, Data Selfie «tiene como objetivo proporcionar una perspectiva personal sobre la minería de datos, el análisis predictivo y nuestra identidad de datos en línea, incluida la información deducida de nuestro consumo. En nuestra sociedad de datos, los algoritmos y big data están definiendo cada vez más nuestras vidas. Por lo tanto, es importante, especialmente para aquellos que son indiferentes a este problema, ser conscientes del poder e influencia que sus propios datos tienen sobre usted».

\section{Biografía de datos}

De gran popularidad en la literatura contemporánea, la biografía nos narra la vida de una determinada persona, "clasificada como un tipo o subgénero literario-histórico, situado dentro de los géneros memorialísticos, a su vez integrados en los ensayísticos» (Aullón de Haro 1992). Nacida como género literario moderno en el Renacimiento a causa de su antropocentrismo y laicismo, fue en el siglo xIx cuando el Romanticismo dio a la biografía dos direcciones diferentes: profundizar en la vida psíquica del biografiado o buscar, por encima de todo, la amenidad literaria, sacrificando la verdad del personaje.
«Más adelante, el Realismo asumió la metodología del Positivismo y acrecentó el número y verificabilidad de las referencias, produciendo una gran búsqueda de documentación sobre el personaje (manuscritos, diarios, correspondencia, testimonios etc.), como elementos fundamentales de la narración biográfica, a fin de situar al biografiado en su auténtico medio histórico-social» (Olmo Ibáñez 2015).

El interés de esta investigación se basa en, a partir de la vertiente heredada de la tradición realista-positivista de la narración biográfica, preguntarnos si se puede realizar, a día de hoy, una biografía coherente a partir de los datos y al acceso a la documentación existente en las redes. En la época del dataceno, podríamos afirmar a priorique nos encontramos en el momento perfecto para desarrollar el modelo biográfico realista, debido a la inmensa información acumulada en el big data. Esta aparente objetividad, donde la información en principio se nos muestra como un recurso óptimo para trazar el relato biográfico, del cual podemos posteriormente, utilizando algoritmos predictivos, inteligencia artificial y análisis semánticos (entre otros métodos computacionales), extraer y generar resultados relativos a perfiles psicológicos, deseos, comportamiento, etc., además de tener información precisa de la ubicación y lugares que el biografiado ha visitado.

Pero esta visión es cuanto menos simplista, ya que el registro cuantitativo de nuestras acciones puede ser sesgado, parcial e inducir a interpretaciones erróneas sobre la vida del sujeto a biografiar (Glauner, Valtchev y State 2018). De mismo modo que el criticado eslogan «conoce tus números para conocerte a ti mismo» del Quantified Self(Reigeluth 2014), nos promete una mejora en la salud y el bienestar personal a través del seguimiento intensivo de nuestros datos biométricos, las biografías de datos nos pueden mostrar una interpretación reduccionista y sesgada del biografiado.

Un claro ejemplo es la obra Annual Reports, de Nicholas Felton (Felton 2005), el cual ha elaborado desde el 2005 una serie de informes gráficos anuales a partir de la recopilación de sus rastros digitales, basados en las experiencias cuantificables de su vida. Entendemos estos informes como una visualización de parte de la vida del autor que, al ser representada gráficamente, puede ayudar a crear una biografía parcial del mismo, ya que muestra de forma analítica toda actividad digital, con gran detalle y datos que de otra forma podrían pasar desapercibidos. Pero toda la información que muestra son datos cuantitativos de su actividad digital y deja de lado el análisis más profundo basado en aspectos cualitativos, psicológicos, dejando el resultado final del trabajo a merced de un posible sesgo de los datos recopilados.

Centrándonos en el uso de los rastros digitales como materia prima para crear obras a partir de los mismos, nos gustaría presentar el trabajo Data Portraits (Martyn Dade-Robertson, s. f.), donde los autores realizan una representación abstracta de los mismos, recordando al trabajo de Flight Patterns, anteriormente mencionado, pero realizados a partir de los datos personales a modo de retrato digital. 


\section{artnodes}

Sin animo de transformar los datos captados, Bruno Ministro decidió imprimir todos los datos de sus búsquedas en internet, para enfatizar el valor de esa información y el uso comercial empresas como Google hacen de los mismos. Su obra Collected Works (20082016) (Ministro 2017) es un claro referente de nuestra investigación Data Biography (Boj y Díaz 2017). Collected Works recopila los datos de las búsquedas en internet, pero Data Biography intenta componer una biografía física global con 365 libros impresos en edición única (uno por día), que recopilan el rastro de los datos digitales generados por Clara Boj y Diego Díaz durante todo el año 2017. Si una biografía es la vida de una persona narrada por otra y consigna aquellos aspectos de su vida mas relevantes y todo lo que, a los ojos del relator, puede interesar de la misma, nuestro proyecto trata de realizar una propuesta de diseño especulativo sobre cómo se podría trazar la biografía situada en el momento actual, a partir de los datos captados de nuestro rastro digital en redes sociales, WhatsApp, emails, webs visitadas, búsquedas en google, imágenes, localización gps, etc.

Cada uno de los 365 libros está numerado correlativamente con un total cercano a las cuarenta mil páginas. Ubicados sobre un estante donde se puede leer el título de la obra, cada columna recopila un mes, cada libro un día y cada línea del libro recoge

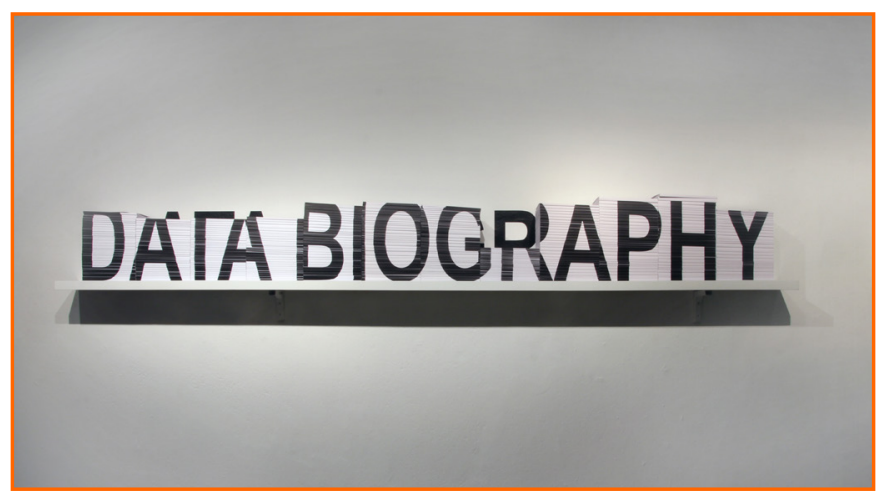

Imagen 4. Data Biography, Clara Boj y Diego Díaz, 2017

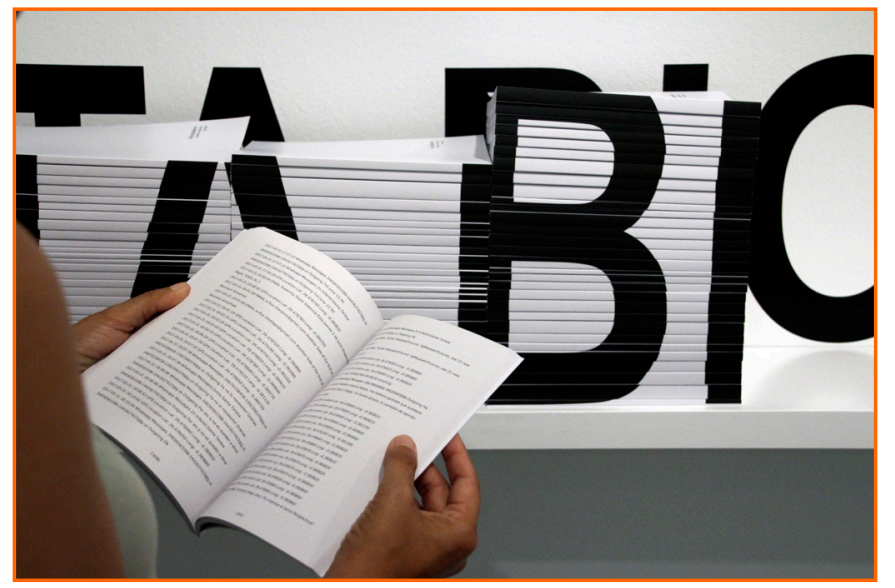

Imagen 5. Data Biography (detalle), Clara Boj y Diego Díaz, 2017 una acción realizada, donde se muestran el registro temporal y los distintos datos de la misma: el tipo de acción (localización GPS, email, WhatsApp, Facebook, Skype, etc) y su contenido. Los vídeos y las fotografías son mostrados en formato imagen, lo que genera un listado de información que permite al visitante acceder a la lectura de todas las acciones realizadas por nosotros durante el año 2017, resaltando que esta información está accesible, al alimentar el inmenso espacio digital del big data.

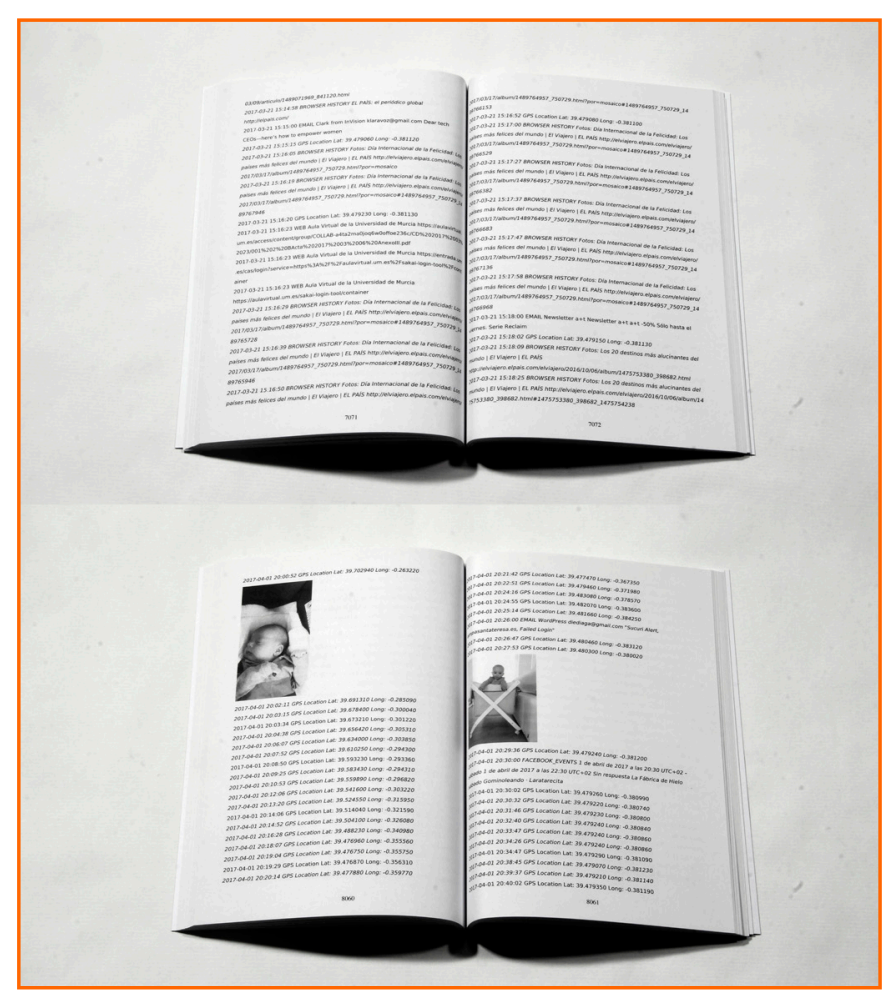

Imagen 6. Data Biography (detalle), Clara Boj y Diego Díaz, 2017

Si realizamos un primer análisis cuantitativo de nuestra actividad a partir de los datos recopilados, podemos visualizar determinadas métricas de nuestra actividad digital, como el número de registros por horas, día y mes, por categoría, etc., que nos muestran los momentos de máxima y mínima actividad digital. Esta información es valiosa, pero ciertamente limitada, pues no nos acerca a una aproximación más rica y pluridimensional de nuestras acciones.

Con el objetivo de cuestionar las herramientas actuales de análisis híbrido cuantitativo-cualitativo de la información, estamos utilizando técnicas de inteligencia artificial como el procesamiento natural de lenguaje y el análisis de imágenes aplicado a distintas categorías. A continuación, describimos brevemente dichos análisis:

- Ubicaciones gps: ubicaciones más visitadas, tipos de desplazamientos, media de distancia recorrida según el tipo de 


\section{artnodes}

http://artnodes.uoc.edu

Prácticas artísticas en la época del dataceno...

desplazamiento, distancia máxima y mínima recorrida según tipo de desplazamiento, tiempo utilizado en cada tipología de desplazamiento, análisis de calorías quemadas diariamente, índice de peligro gastrovascular según actividad física asociada al desplazamiento realizado diariamente. Etiquetado y clasificación por tipologías de lugares visitados.

- Textos: media, número máximo y mínimo de palabras escritas diariamente, reconocimiento de conceptos más utilizados, análisis de sentimientos. Análisis y clasificación de comunicaciones (personas más y menos contactadas). Medios de comunicación más utilizados. Fuentes de información más consultadas.

- Imágenes y vídeos: clasificación colorimétrica, etiquetado y clasificación automática de imágenes, detección de rostros y clasificación de estados de ánimo en base a los rostros detectados. Clasificación de rostros por genero, edad y tipo de actividad.

- Páginas webs visitadas: número de páginas visitadas por horas, días y meses, etiquetado y clasificación de las mismas. Fuentes de información más consultadas.

Estamos realizando todos estos análisis con herramientas disponibles en la red y también utilizamos distintas API de código abierto. Nuestro objetivo final es el de realizar una reflexión crítica sobre la utilidad y neutralidad de las herramientas disponibles, verificar el posible sesgo en la muestra para la generación de los datasets y cuestionar la utilidad de los algoritmos de extracción y análisis de datos personales. En base a los primeros resultados obtenidos, ${ }^{1}$ aunque no son concluyentes, podemos afirmar que actualmente es posible establecer un perfil definido de modelos de comportamiento y psicológico de las personas analizadas, que suelen tener un alto índice de error y resultados bastante subjetivos, pero que debido a la importancia de los datos en la época del dataceno son difícilmente refutables y, por lo tanto, nos pueden acercar hacia un futuro distópico ciertamente preocupante.

\section{Referencias}

Albino, Vito; Umberto Berardi y Rosa Maria Dangelico. 2015. «Smart Cities: Definitions, Dimensions, Performance, and Initiatives». Journal of Urban Technology. https://doi.org/10.1080/1063073 2.2014.942092

Asri, Hiba; Hajar Mousannif, Hassan Al Moatassime et al. 2015. «Big Data in Healthcare: Challenges and Opportunities». En Proceedings of 2015 International Conference on Cloud Computing Technologies and Applications, CloudTech 2015. https://doi. org/10.1109/CloudTech.2015.7337020.
Aullón de Haro, Pedro. 1992. Teoría Del Ensayo Como Categoría Polémica y Programática En El Marco de Un Sistema Global de Géneros. Editorial Verbum.

Balebako, Rebecca; Jaeyeon Jung, Wei Lu et al. 2013. «'Little Brothers Watching You': Raising Awareness of Data Leaks on Smartphones». En Proceedings of the Ninth Symposium on Usable Privacy and Security. https://doi.org/10.1145/2501604.2501616.

Boj, Clara y Diego Diaz. 2017. «Data Biography». 2017. http://www. lalalab.org/data-biography/.

Boj, Clara, Diego Díaz, Pau Alsina et al. 2010. Observatorio. Diputación de Valencia, Sala Parpalló.

Callanan, Martin John. 2015. «I Cannot Not Communicate». 2015. http://greyisgood.eu/communicate/

Chen, Yiheng and Dawei Han. 2018. «Water Quality Monitoring in Smart City: A Pilot Project». Automation in Construction. https:// doi.org/10.1016/j.autcon.2018.02.008

Colombo, Pietro and Elena Ferrari. 2015. «Privacy Aware Access Control for Big Data: A Research Roadmap». Big Data Research. https://doi.org/10.1016/j.bdr.2015.08.001

Curnutt, John L. 2007. «A Guide to the Homogenocene». Ecology. https:// doi.org/10.1890/0012-9658(2000)081[1756:agtth]2.0.c0;2.

D3. 2011. «D3.Js». 2011. https://d3js.org/

Danaher, John. 2016. «The Threat of Algocracy: Reality, Resistance and Accommodation». Philosophy \& Technology 29 (3): 245-68. https://doi.org/10.1007/s13347-015-0211-1

Diamantoulakis, Panagiotis D.; Vasileios M. Kapinas y George K. Karagiannidis. 2015. «Big Data Analytics for Dynamic Energy Management in Smart Grids». Big Data Research. https://doi. org/10.1016/j.bdr.2015.03.003

Diaz, Diego, Clara Boj, A.D. Cheok et al. 2005. «Free Networks Visible Networks». En Proceedings of the 2005 International Conference on Active Media Technology. Vol. 2005. https://doi.org/10.1109/ AMT.2005.1505404

Erevelles, Sunil; Nobuyuki Fukawa and Linda Swayne. 2016. «Big Data Consumer Analytics and the Transformation of Marketing». Journal of Business Research. https://doi.org/10.1016/j.jbusres.2015.07.001

Felton, Nicholas. 2005. «Feltron». 2005. http://feltron.com/index.html. Frick, Laurie, and Thirteen23. 2014. «FRICKbits». 2014. http://www. frickbits.com/.

Friendly, M. 2006. «A Brief History of Data Visualization». En Handbook of Computational Statistics: Data Visualization, editado por C. Chen, W. Härdle y A. Unwin, III:15-56. Heidelberg: Springer-Verlag. Furht, Borko y Flavio Villanustre. 2016. «Introduction to Big Data». En Big Data Technologies and Applications. https://doi. org/10.1007/978-3-319-44550-2_1

\footnotetext{
1. El análisis y presentación en detalle de estos resultados escapa a los límites e intereses de esta publicación.
} 


\section{artnodes}

Glauner, Patrick; Petko Valtchev y Radu State. 2018. «Impact of Biases in Big Data». En Proceedings of the 26th European Symposium on Artificial Neural Networks, Computational Intelligence and Machine Learning (ESANN 2018).

Graeff, Timothy R. y Susan Harmon. 2002. «Collecting and Using Personal Data: Consumers' Awareness and Concerns". Journal of Consumer Marketing. https://doi.org/10.1108/07363760210433627

Guljajeva, Varvara y Mar Canet. 2017. «Data Shop». 2017. http://www. varvarag.info/data-shop/.

Haraway, Donna. 2015. «Anthropocene, Capitalocene, Plantationocene, Chthulucene: Making Kin». Environmental Humanities 6 (1):159-65. https://doi.org/10.1215/22011919-3615934

IDC. 2018. «IDC FutureScape: Worldwide IT Industry 2018 Predictions». IDC FutureScape.

Ishii, Hiroshi; David Lakatos, Leonardo Bonanni et al. 2012. «Radical Atoms: Beyond Tangible Bits, Toward Transformable Materials». Interactions. https://doi.org/10.1145/2065327.2065337

Jucevicius, Robertas, Irena Patasienè y Martynas Patasius. 2014. «Digital Dimension of Smart City: Critical Analysis». Procedia - Social and Behavioral Sciences. https://doi.org/10.1016/j. sbspro.2014.11.137

KesselsKramer. 2011. «KK: 24 HRS IN PHOTOS». 2011. http://www. kesselskramer.com/exhibitions/24-hrs-of-photos

Kim, Gang-Hoon; Silvana Trimi y Ji-Hyong Chung. 2014. «Big-Data Applications in the Government Sector». Communications of the ACM. https://doi.org/10.1145/2500873

Kitchin, Rob. 2014. «Big Data, New Epistemologies and Paradigm Shifts». Big Data \& Society. https://doi.org/10.1177/2053951714528481

Koblin, Aaron. 2009. «Flight Patterns». 2009. http://www.aaronkoblin. com/work/flightpatterns/

Lima, Manuel. 2005. «A Visual Exploration on Mapping Complex Networks». 2005. http://www.visualcomplexity.com/vc/

Malhi, Yadvinder. 2017. «The Concept of the Anthropocene». Annual Review of Environment and Resources. https://doi.org/10.1146/ annurev-environ-102016-060854

Martyn Dade-Robertson, Karamba. (s. f.). «Data Portraits». 2013. http://www.dataportraits.co.uk/

Mashey, John R. 1998. «Big Data and the next Wave of InfraS-Tress». Computer Science Division Seminar, University of California, Berkeley. https://doi.org/10.1007/978-3-658-11589-0

McCandless, David. 2009a. «Information Is Beautiful». Also Published as The Visual Miscellaneum: A Colorful Guide to the World's Most Consequential Trivia.

McCandless, David. 2009b. «Information Is Beautiful». 2009. https:// informationisbeautiful.net/

Ministro, Bruno. 2017. «Collected Works». 2017. https://projectocandonga.wordpress.com/trabalhos/collected-works/
Moore, Jason W. 2016. «Introduction. Anthropocene or Capitalocene. Nature, History and the Crisis of Capitalism». Anthropocene or Capitalocene. Nature, History and the Crisis of Capitalism. https:// doi.org/10.1017/CB09781107415324.004

Olmo Ibáñez, María Teresa del. 2015. Teoría de La Biografía. Dykinson. https://www.dykinson.com/libros/teoria-de-la-biografia/9788490855768/

Ortiz, Santiago. 2002. «Moebio Labs I Drumwave». 2002. http:// moebio.com/.

Philip Chen, C. L. y Chun Yang Zhang. 2014. «Data-Intensive Applications, Challenges, Techniques and Technologies: A Survey on Big Data». Information Sciences. https://doi.org/10.1016/j. ins.2014.01.015

Reigeluth, Tyler Butler. 2014. «Why Data Is Not Enough: Digital Traces as Control of Self and Self-Control». Surveillance \& Society 12 (2): 243-54. https://doi.org/10.24908/ss.v12i2.4741

Roth, Evan. 2012. «Internet Cache Self Portrait». 2012. http://www. evan-roth.com/work/internet-cache-self-portrait/

Samways, M. J. 1999. «Translocating Fauna to Foreign Lands: Here Comes the Homogenocene». Journal of Insect Conservation 3 (2): 65-66.

Schrems, Max. 2011. «Europe-v-Facebook.Org I EUROPE versus FACEBO0K». 2011. http://europe-v-facebook.org/EN/en.html

Schwartz, Paul M. 2004. «Property, Privacy, and Personal Data». Harvard Law Review. Vol. 117, Vol.7, p. 2055. https://papers. ssrn.com/sol3/papers.cfm?abstract_id=721642

Smith, Matthew, Christian Szongott, Benjamin Henne et al. 2012. «Big Data Privacy Issues in Public Social Media». IEEE International Conference on Digital Ecosystems and Technologies. https://doi. org/10.1109/DEST.2012.6227909

Sosa, Ricardo, Victoria Gerrard, Antonio Esparza et al. 2018. «Data Objects: Design Principles For Data Physicalisation». En International Design Conference. https://doi.org/10.21278/idc.2018.0125

Thi Duc, Hang Do; Regina Flores Mir Leon Eckert et al. 2016. «Data Selfie». 2016. https://dataselfie.it/\#/.

Vicente, José Luis de.; Honor Harger and Josep Perelló. 2011. Invisible Fields: Geographies of Radio Waves. Arts Santa Mònica.

Vicente, Jose Luis De and Olga Subirós. 2014. «Big Bang Data I CCCB». 2014. http://bigbangdata.cccb.org/es/.

Weaver, Stephen D.; Mark Gahegan. 2010. «Constructing, visualizing, and analyzing a digital footprint*». Geographical Review. https:// doi.org/10.1111/j.1931-0846.2007.tb00509.x

Zalasiewicz, Jan; Mark Williams, Alan Haywood et al. 2011. «The Anthropocene:A New Epoch of Geological Time?».- Philosophical Transactions of the Royal Society A: Mathematical, Physical and Engineering Sciences. https://doi.org/10.1098/rsta.2010.0339 


\section{artnodes}

http://artnodes.uoc.edu

Prácticas artísticas en la época del dataceno...

\section{CV}

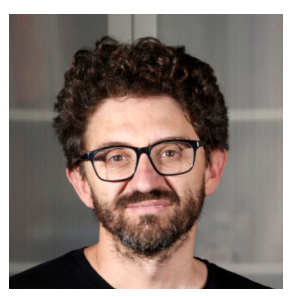

\section{Diego Díaz}

Universitat Juame I

daz@uji.es

Departamento de Ingeniería de Sistemas Industriales y Diseño

Universidad Jaume I

12071 Castelló de la Plana, España

Profesor de la Universidad Jaume I, donde imparte clases en el grado de Diseño y Desarrollo de Videojuegos. Durante 2004 y 2006 fue Research Associated en el Interaction and Entertainment Research Center de la Nanyang Technological University, Singapur. Junto a Clara Boj, sus investigaciones han sido presentadas internacionalmente en centros de reconocido prestigio y revistas indexadas. Han disfrutado de residencias de creación e investigación en instituciones como Hangar en Barcelona, Interface Culture Lab en Linz (Austria), Mixed Reality Lab (Singapur), Symbiotic System Lab en Kyoto (Japón). Entre otras distinciones han sido premiados con la Beca de Investigación Alfons Roig de la Diputación de Valencia en 2006 y con el Incentivo a la producción en la competición internacional Vida 13.2 Arte y Vida Artificial. Actualmente desarrollan, junto a otros investigadores, el proyecto de investigación «Reset Mar Menor: Laboratorio de imaginarios para un paisaje en crisis», financiado por la Fundación Carasso en su convocatoria de Arte Ciudadano. 


\section{artnodes}

http://artnodes.uoc.edu

Prácticas artísticas en la época del dataceno...

\section{CV}

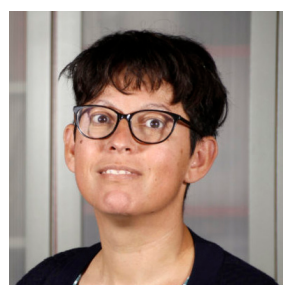

\section{Clara Boj}

Universidad Politécnica de Valencia

claboto@esc.upv.es

Departamento de Escultura

Universidad Politécnica de Valencia

46022 Valencia, España

Profesora de la Universidad Politécnica de Valencia. Durante 2004 al 2006 fue Research Fellow en el Interaction and Entertainment Research Center de la Nanyang Technological University, Singapur. Junto a Diego Diaz sus investigaciones han sido presentadas internacionalmente en centros de reconocido prestigio y revistas indexadas. Han disfrutado de residencias de creación e investigación en instituciones como Hangar en Barcelona, Interface Culture Lab en Linz (Austria), Mixed Reality Lab (Singapur), Symbiotic System Lab en Kyoto (Japón). Entre otras distinciones han sido premiados con la Beca de Investigación Alfons Roig de la Diputación de Valencia en 2006 y con el Incentivo a la producción en la competición internacional Vida 13.2 Arte y Vida Artificial.

Actualmente desarrollan, junto a otros investigadores, el proyecto de investigación «ResetMar Menor: Laboratorio de imaginarios para un paisaje en crisis», financiado por la Fundación Carasso en su convocatoria de Arte Ciudadano.

En el ámbito de la mediación artística, en el que Clara Boj obtuvo su doctorado en el año 2003, actualmente es codirectora del Master Permea, organizado por el Consorcio de Museos de la GeneralitatValenciana y de la Universitat de Valencia y dirige el proyecto transversalia. net, organizado por la Generalitat Valencia y el Servicio de Formación de Profesorado (CEFIRE) de la Generalitat Valenciana. 